\title{
Hepatocellular Carcinoma in Acute Intermittent Porphyria: Incidental or Expected?
}

\author{
Mary Mathews ${ }^{1, *}$, Lauren Paish ${ }^{2}$, Abhishek Kumar $^{3}$, Nazia Khan ${ }^{4}$, Michael Maroules ${ }^{3}$ \\ ${ }^{1}$ Department of Internal Medicine, St. Joseph’s Regional Medical Center, New York Medical College \\ ${ }^{2}$ Medical Student, New York Medical College \\ ${ }^{3}$ Division of Hematology Oncology, Department of Internal Medicine, St. Joseph's Regional Medical Center \\ ${ }^{4}$ Department of Internal Medicine, St. Joseph’s Regional Medical Center, New York Medical College \\ *Corresponding author: mary.mathews@live.ca
}

Received April 07, 2015; Revised April 17, 2015; Accepted April 23, 2015

\begin{abstract}
Acute intermittent porphyria (AIP) is a rare genetic disorder involving an insufficiency of the enzyme porphobilinogen deaminase of the heme biosynthesis pathway. Clinically, this manifests as acute attacks of abdominal pain and neuropsychiatric dysfunction, at times these attacks can be life threatening. Despite an autosomal dominant pattern of inheritance, only a minority develop acute attacks due to poor penetrance, thus representing the manifest and latent type of AIP. A unique association between AIP and Hepatocellular carcinoma (HCC) has been reported by some studies recommended screening recommendations for AIP gene carriers over the age of 50. We report a case of 55 year old Slovakian female with a past medical history of acute intermittent porphyria. She presented to the emergency room complaining of persistent, sharp, right upper quadrant pain with intractable nausea and vomiting. A diagnosis of acute episode of AIP was made, along with incidental finding of hepatocellular carcinoma.
\end{abstract}

Keywords: acute intermittent porphyria, porphobilinogen deaminase, hepatocellular carcinoma

Cite This Article: Mary Mathews, Lauren Paish, Abhishek Kumar, Nazia Khan, and Michael Maroules, "Hepatocellular Carcinoma in Acute Intermittent Porphyria: Incidental or Expected?." American Journal of Medical Case Reports, vol. 3, no. 5 (2015): 141-143. doi: 10.12691/ajmcr-3-5-6.

\section{Introduction}

Acute intermittent porphyria (AIP) is a rare genetic disorder with autosomal dominant disorder involving an insufficiency of porphobilinogen deaminase enzyme of the heme biosynthesis pathway, it clinically results in acute attacks of abdominal pain and neuropsychiatric dysfunction and at times can lead to life threatening outcomes. [1,2,11] Despite an autosomal dominant pattern of inheritance is present, only a minority of carriers develop acute attacks due to poor penetrance, thus representing the manifest and latent type of AIP within this population. [3] There is a reported incidence of AIP is 0.13 and 0.51 cases per year per million inhabitants in European countries and in Sweden respectively [3,4].

A unique correlation between AIP and Hepatocellular carcinoma was first described by Lithner and Wetterberg in 1984 amongst the Swedish population, with recent studies reporting an incidence per year of HCC to be $0.8 \%$ in genetic carriers over 50 years of age. [5,7] In a prospective study involving 650 French carriers of acute hepatic porphyrias, AIP gene carriers were at increased risk for HCC compared to the french population. [3,8] This reported correlation between AIP and HCC is very intriguing, and is a possible long term complication of AIP reported in both symptomatic and asymptomatic carriers [3].
We report a case of a Slovakian woman, who presented with an acute attack of AIP, and was incidentally found to have HCC. We plan to discuss the various reported correlations between AIP and HCC, the possible mechanisms leading to the development of HCC and the need for screening recommendations within this population.

\section{Case Report}

A 55 year old Slovakian female with a past medical history of acute intermittent porphyria diagnosed over 25 years ago presented to the emergency room complaining of sharp, constant right upper quadrant pain and intractable nausea and vomiting.

Her vital signs on presentation: Temperature-97.9 F, Blood Pressure-176 /98 mmHg, pulse-98, respiratory rate $-18 /$ min, pulse Oximetry-98-100\% on room air. On physical examination she appeared dehydrated and had right upper quadrant tenderness with an absence of murphy's sign, the abdomen was soft with bowel sounds and no organomegaly was appreciated.. Laboratory studies revealed a hemoglobin-14.6 g/dL, hematocrit-43.8 \%, white blood cell count $-13.8 \mathrm{~K} / \mathrm{mm} 3$, platelets- $147 \mathrm{~K} / \mathrm{mm} 3$. Acid base balance showed metabolic alkalosis with bicarbonate level -28 MEQ/L. Renal functions revealed a BUN/ creatinine ratio of $27 \mathrm{mg} / \mathrm{dL} / 0.8 \mathrm{mg} / \mathrm{dL}$, likely 
secondary to dehydration. Liver function tests showed a AST-33 U/L, ALT-23 U/L, alkaline phosphatase-78 IU/L, total bilirubin-0.4 and albumin-4.4 G/DL. Urine porphobilinogen levels were found to be significantly elevated at $43.3 \mathrm{mg} / \mathrm{L}$ (normal value: $0-2 \mathrm{mg} / \mathrm{L}$ ). Computed tomography scan (CT scan) of the abdomen, revealed a large fungating mass involving the right upper lobe of the liver with adjacent satellite lesions, these findings were highly suspicious for Hepatocellular Carcinoma (HCC). A triple phase CT scan of abdomen Figure 1, confirmed the presence of HCC with arterial enhancement of the mass measuring $9.5 \mathrm{~cm} \mathrm{x} 8.7 \mathrm{~cm} \mathrm{x}$ $6.3 \mathrm{~cm}$ with adjacent satellite lesions predominantly in the right lobe of the liver (involving segments $4 \mathrm{a}$ and 8), borderline splenomegaly and no portal vein thrombosis.
The serum alpha fetal protein level was found to be only 3 $\mathrm{ng} / \mathrm{ml}$. HIV, Hepatitis A, B, and C serological tests were negative. In the acute setting, patient was aggressively treated with glucose loading and intravenous fluids. Antihypertensive and supportive therapy was given during the hospital course. She showed significant clinical improvement, sparing the need to begin hemin therapy. She received selective chemoembolization to segments 4a and 8 involving the hepatocellular carcinoma, as resection of this large tumor required extended right hepatic lobectomy which was considered very high risk in our patient. A follow up CT scan done a month later revealed reduction in dominant mass size to $7.3 \mathrm{~cm}$ and decrease in satellite lesions as well; she is scheduled to receive subsequent sessions of chemoembolization.

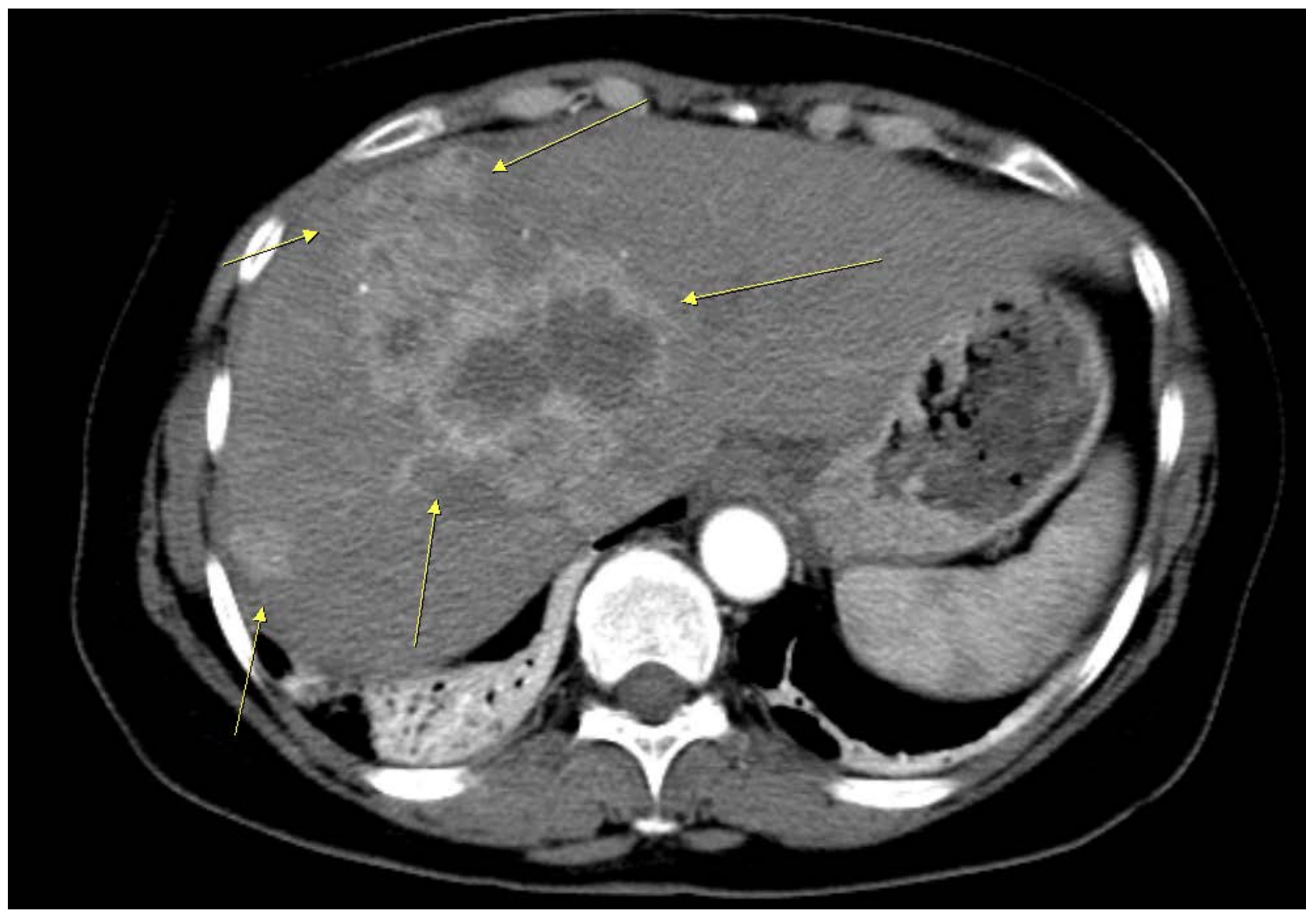

Figure 1. Triple phase CT indicating large hepatocellular carcinoma with satellite lesions

\section{Discussion}

In porphyria there is a reduction in free heme levels along with auto-oxidation of delta-aminolevulinic acid that can lead to increased free radicals, increasing the mutagenic potential that could lead to injury and development of HCC. [3,6] An enzyme deficient in AIP, urophorphyrinogen-1-synthetase (also known as porphobilinogen deaminase) is also linked to a mutation on chromosome 11 that may cause transformation of this gene into an oncogene for HCC. [1,5,9,10] Another reported pathophysiological correlation is the deletion of $11 p$ found in patients with hepatitis $\mathrm{B}$ that progress to develop HCC. [1,9] There is also support for the theory that porphyrins are carcinogenic themselves. [10] Despite these proposed hypotheses on the pathogenesis of HCC in patients with AIP the exact pathogenesis remains unknown.

Amongst AIP gene carriers with no other risk factors for HCC, the progression to HCC was found to be more prevalent in females and elderly patients. [1,6,7] It was also noted to be more common in patients who actively manifested AIP episodes than the latent form, this perhaps can be attributed to the cumulative effect of interrupted heme synthesis and damage caused by free radicals on hepatic tissues. [6,7] Liver cirrhosis was also found to be predominantly in AIP patients (12\%) than controls (0.5\%). [6] The annual incidence of HCC in AIP gene carriers ( $>50$ years) is reported to be $0.8 \%$ in Sweden, $0.16 \%$ in a French study and $0.3 \%$ in a Switzerland study. [6,7] The marked incidence demonstrated in Sweden is most likely due to a greater population of AIP gene carriers with a 
predominant founder mutation W198X. [3,6] This significantly increased risk for HCC has been more frequently reported in genetic carriers of AIP compared to other forms of hepatic porphyrias [3].

Although there are several European studies reporting the incidence and prevalence of HCC amongst the AIP gene population, there is a clear need for robust epidemiological studies indicating the current incidence and prevalence of AIP amongst European immigrants within the United States. This population represented 12 percent of all immigrants 4.8 million of the country's 40 million immigrants in 2010 [12].

In recent years the incidence of HCC has tripled in the United States, although surveillance is recommend for high risk groups such as in HCV, HBV carriers, European studies have recommended that AIP be added to list if risk factors at least in patients diagnosed with HCC. [7,13] Annual ultrasound screening is a recommendation made by these studies to be performed for AIP gene carriers a proven history of AIP, starting at 50 years of age. [3,6,7] There was also no reported benefit in using liver function tests and alpha- feto protein level as screening tools for HCC $[3,6,7]$.

Considering the rarity of AIP in the United States, diagnosing an acute attack becomes challenging as only a minority of genetic carriers become acutely symptomatic, especially since these symptoms mimic other common medical conditions. HCC and Acute AIP attacks may present similarly, thereby delaying diagnosis of HCC in an established manifest AIP patient. [1,3,4] Through this case report, we would like to emphasize the increased risk of HCC in AIP gene carriers, physicians in western world need to be aware of this reported correlation and take the appropriate measures needed while managing their care. Future studies clarifying the relationship and underlying pathogenesis of AIP leading to HCC in a genetically heterogenous population is paramount to preventing some morbidity and mortality caused by this rare disorder.

\section{References}

[1] Kauppinen, R., \& Mustajoki, P. (1988). Acute hepatic porphyria and hepatocellular carcinoma. British journal of cancer, 57(1), 117

[2] Linet, M. S., Gridley, G., Nyrén, O., Mellemkjaer, L., Olsen, J. H., Keehn, S., ... \& Fraumeni, J. F. (1999). Primary liver cancer, other malignancies, and mortality risks following porphyria: a cohort study in Denmark and Sweden.American journal of epidemiology, 149(11), 1010-1015.

[3] Stewart, M. F. (2012). Review of hepatocellular cancer, hypertension and renal impairment as late complications of acute porphyria and recommendations for patient follow-up. Journal of clinical pathology, 65(11), 976-980.

[4] Elder, G., Harper, P., Badminton, M., Sandberg, S., \& Deybach, J. C. (2013). The incidence of inherited porphyrias in Europe. Journal of inherited metabolic disease, 36(5), 849-857.

[5] Lithner, F., \& Wetterberg, L. (1984). Hepatocellular carcinoma in patients with acute intermittent porphyria. Acta Medica Scandinavica, 215(3), 271-274.

[6] Andersson, C., Bjersing, L., \& Lithner, F. (1996). The epidemiology of hepatocellular carcinoma in patients with acute intermittent porphyria. Journal of internal medicine, 240(4), 195201.

[7] Innala, E., \& Andersson, C. (2011). Screening for hepatocellular carcinoma in acute intermittent porphyria: a 15 - year follow - up in northern Sweden. Journal of internal medicine, 269(5), 538-545.

[8] Andant, C., Puy, H., Faivre, J., \& Deybach, J. C. (1998). Acute hepatic porphyrias and primary liver cancer. New England Journal of Medicine, 338(25), 1853-1854.

[9] Rogler, C. E., Sherman, M., Su, C. Y., Shafritz, D. A., Summers, J., Shows, T. B., ... \& Kew, M. (1985). Deletion in chromosome 11p associated with a hepatitis B integration site in hepatocellular carcinoma. Science, 230(4723), 319-322.

[10] Bengtsson, N. O., \& Hardell, L. (1986). Porphyrias, porphyrins and hepatocellular cancer. British journal of cancer, 54(1), 115.

[11] Jeans, J. B., Savik, K., Gross, C. R., Weimer, M. K., Bossenmaier, I. C., Pierach, C. A., \& Bloomer, J. R. (1996). Mortality in patients with acute intermittent porphyria requiring hospitalization: a United States case series.American journal of medical genetics, 65(4), 269-273.

[12] Russell, A. and Batalova,J. (2012).European Immigrants in the United States. Retrieved from http://http://www.migrationpolicy.org.

[13] Altekruse, S. F., McGlynn, K. A., \& Reichman, M. E. (2009). Hepatocellular carcinoma incidence, mortality, and survival trends in the United States from 1975 to 2005. Journal of Clinical Oncology, 27(9), 1485-1491. 